\title{
A NOVEL THEORETICAL MODELING FOR PREDICTING THE SOUND ABSORPTION OF WOVEN FABRICS USING MODIFICATION OF SOUND WAVE EQUATION AND GENETIC ALGORITHM
}

\author{
Valentinus Galih Vidia Putra ${ }^{1,{ }^{\star}}$, Irwan ${ }^{1}$, Andrian Wijayono $^{2}$, JulianyNingsih Mohamad ${ }^{3}$, Yusril Yusuf ${ }^{4}$ \\ 1 Department of Textile Engineering, Politeknik STTT Bandung, Bandung, Indonesia \\ 2 Department of Textile and Apparel Engineering, Politeknik STTT Bandung, Bandung, Indonesia \\ 3 Department of Physics, Universitas Nusa Cendana, Kupang, Indonesia \\ 4 Department of Physics, Universitas Gadjah Mada, Yogyakarta, Indonesia \\ ${ }^{\star}$ Corresponding author. E-mail: valentinus@kemenperin.go.id
}

Abstract:

Woven fabric in Indonesia is generally known as a material for making clothes and it has been applied as an interior finishing material in buildings, such as sound absorbent material. This study presents a new method for predicting the sound absorption of woven fabrics using a modification of the wave equations and using genetic algorithms. The main aim of this research is to study the sound absorption properties of woven fabric by modeling using a modification of the sound wave equations and using genetic algorithms. A new model for predicting the sound absorption coefficient of woven fabric (plain, twill 2/1, rips and satin fabric) as a function of porosity, the weight of the fabric, the thickness of the fabric, and frequency of the sound wave, was determined in this paper. In this research, the sound absorption coefficient equation was obtained using the modification of the sound wave equation as well as using genetic algorithms. This new model included the influence of the sound absorption coefficient phenomenon caused by porosity, the weight of the fabric, the thickness of fabric as well as the frequency of the sound wave. In this study, experimental data showed a good agreement with the model

Keywords:

Acoustic, Sound absorption Coefficient, woven fabric

\section{Introduction}

The application of theoretical physics in textile science, especially in computational physics and material physics is widely found both experimentally and theoretically. One of the applications of physic in textile science is to produce woven fabrics that can be used as sound-absorbing materials. Researchers examined the application of woven fabrics as sound-absorbent materials both experimentally and theoretically. [1, 2, 3, 4, 5, 6, 7, 8, 9, 10, 11]. The utilization of woven fabric as a material that has the potential to be used as a sound absorber material was reported by Shoshani and Rosenhouse [1]. Several studies have been conducted to utilize woven fabrics as soundabsorbent materials, and there have some efforts to measure the sound absorption coefficient of the woven fabric $[12,13,14]$. Some researchers reported $[12,15,16]$ that woven fabrics have several micro-holes (around 1 $\mathrm{mm}$ ) where the textile fabric can be assumed as the microperforated panel (MPP) which has quite a lot of porosity. Several studies have shown that in the formation of textile woven fabrics as sound-absorbent materials, factors such as porosity, fabric thickness, fabric surface density, 
and frequency affect the sound absorption coefficient on the fabric $[1,12,13,14,17,18,19]$. Research on acoustic textiles both experimentally and theoretically has been done in the area of nonwoven acoustic fabrics, but in-depth studies of woven and knit fabrics are still very limited. Theory and modeling studies on the properties of weight, thickness, porosity, and frequency of sound waves on woven fabrics are still rarely found as a complete and structured formula $[20,21,22,23,24]$. Several researchers $[1,12,13,14,17,18,19,23]$ had investigated the relationship between the impedance and the absorption characteristics of fabric as well as the effects of thickness, weight, and fabric. Vassiliadis [25] proposed that the sound absorption coefficient can be formulated using the following Eq. (1)

$$
a_{a}=1-\left(\frac{Z_{2}-Z_{1}}{Z_{2}+Z_{1}}\right)^{2}=1-R^{2}
$$

Where the term $a_{a}$ is the sound absorption coefficient; $Z_{2}$, the acoustic surface impedance on the media 2 in the unit $\left(\mathrm{kg} / \mathrm{m}^{2} \mathrm{~s}\right.$ or rayl); $Z_{1}$, the acoustic surface impedance on the media 1 in the unit $\left(\mathrm{kg} / \mathrm{m}^{2} \mathrm{~s}\right.$ or rayl); $R$, the reflection factor or pressure reflection coefficient. Another model to measure the total sound absorption coefficient was proposed by Yairi, Sakagami, Takebayashi, and Morimoto [26] and it can be expressed as Eq. (2)

$$
a_{a}=\frac{4 \operatorname{Re}\left\{Z_{t o t}\right\}}{\left(1+\operatorname{Re}\left\{Z_{t o t}\right\}\right)^{2}+\left(\operatorname{Img}\left\{Z_{t o t}\right\}\right)^{2}}
$$

Where the term $a_{a}$ is the sound absorption coefficient; $Z_{t o t}$, the total acoustic surface impedance on the media in the unit $\left(\mathrm{kg} / \mathrm{m}^{2} \mathrm{~s}\right.$ or rayl). According to Prasetiyo, et al. [27], the sound absorption coefficient can be written using the following Eq. (3)

$$
\alpha=\frac{4 \operatorname{Re}\left\{Z_{t o t}\right\}}{\left(1+\operatorname{Re}\left\{Z_{t o t}\right\}\right)^{2}}=1-\left(\frac{Z_{2}-\rho_{0} c_{0}}{Z_{2}+\rho_{0} c_{0}}\right)^{2}
$$

Where the term $\alpha$ and $Z_{2}$ are the sound absorption coefficient and the acoustic impedance; $Z_{t o t}$, the total acoustic surface impedance on the media in the unit $\left(\mathrm{kg} / \mathrm{m}^{2} \mathrm{~s}\right.$ or rayl); $\rho_{o}$, the density of air; $c_{o}$, the speed of acoustic wave. According to Vassiliadis [25] and $\mathrm{Na}$ et al. [23], the sound absorption coefficient of fabric is influenced by several factors such as porosity, fabric thickness, and the type of fiber in the fabric. Some researchers $[1,12,13,14$, $17,18,19,23,25,26,27,28,29]$ concluded that: 1) the sound absorption coefficient increases with a decrease in yarn diameter; 2) The shape of the structure of woven fabric affects the value of sound absorption coefficient, plain woven fabric has a better fabric structure in increasing the value of sound absorption coefficient compared to other types of woven fabric (Fabrics are woven with equal warp and weft density); 3) the higher the porosity, the lower is the sound absorption coefficient; 4) the higher the volume density of the fabric, the higher is the sound absorption coefficient. In some studies, the value of the sound absorption coefficient is influenced by several factors, such as the value of the speed of acoustic wave or speed of sound, the acoustic pressure, the angular frequency, the wavenumber, particle speed of the propagation medium which vibrates around the development of sound and also the acoustic impedance $[1,12,13,14,17,18,19,23$, 25, 26, 27, 28, 29]. According to Atalla et al. [28] and Maa [4], the acoustic impedance of the sound absorber consists of real parts and imaginary parts (resistance impedance for real parts and reactance impedance for imaginary parts). The impedance of the sound absorber was formulated using the following Eq. (4) to Eq. (6):

$$
\begin{gathered}
Z=Z_{\text {resistance }}+Z_{\text {reactance }}=r+j \omega m \\
Z=r+j \omega m=\frac{32 \eta t}{\rho_{o} c_{o} d^{2} p}\left(\sqrt{1+\frac{x^{2}}{32}}+\frac{x d \sqrt{2}}{8 t}\right) \\
+\frac{j \omega t}{p c_{o}}\left(1+\left(9+\frac{x^{2}}{2}\right)^{-1 / 2}+\frac{0.85 d}{t}\right) \\
x=\frac{d}{2} \sqrt{\frac{\omega \rho}{\eta}}
\end{gathered}
$$

Where the terms $r$ and $m$ are the acoustic resistance and the acoustic reactance, $\rho_{o}$ is the density of air, $c_{o}$ is the speed of acoustic wave, $\omega$ is the angular frequency, $t$ is the thickness of material, $d$ is the diameter of the holes, $p$ is the perforation ratio and $\eta$ is the viscosity of air. Maa [5] reported that the impedance of the sound absorber could be written using the following Eq. (7):

$$
Z=-j \cot \left(\frac{\omega D}{c}\right)
$$

Where the terms $D$ and $\mathrm{c}$ are the cavity depth and the speed of acoustic wave. Vassiliadis [25] formulated the impedance of the sound absorber and the propagation wavenumber as a function of flow resistivity for fibrous porous material using the following Eq. (8):

$$
\begin{gathered}
Z=\rho_{o} c_{o}\left(1+0.0571 X^{-0.754}-j 0.087 X^{-0.732}\right) \\
k=\omega / c_{o}\left(1+0.0978 X^{-0.700}-j 0.189 X^{-0.595}\right) \\
X=\frac{\rho_{o} f}{\sigma}=\frac{\rho_{o} f v d}{P}
\end{gathered}
$$


Where the term $\sigma$ is flow resistivityin the unit $\left(\mathrm{kg} / \mathrm{m}^{2} \mathrm{~s}\right.$ or rayl); $\rho_{o}$ is the density of air, $c_{o}$ is the speed of acoustic wave, $\omega$ is the angular frequency, $d$ is the thickness of the absorbent, $v$ is the mean steady flow velocity and $P$ is pressure drop. Vassiliadis [25] has also formulated the impedance of the sound absorber, $Z$, in simple form using the following Eq. (11)

$$
Z=\rho v
$$

Where the terms $\rho$ and $v$ are the density of sound in specific medium in the unit $\left(\mathrm{kg} / \mathrm{m}^{3}\right)$ and the speed of sound in specific medium in the unit $\left(\mathrm{m} / \mathrm{s}^{2}\right)$. The equation of sound wave can be formulated as, Eq. (12) to Eq. (13) [25]:

$$
\begin{gathered}
\nabla^{2} P-\frac{1}{v^{2}} \frac{d^{2} P}{d t^{2}}=0 \\
\boldsymbol{P}(z, t)=P_{o} \cos (\boldsymbol{k} . \boldsymbol{r}-\omega t) \\
v=\lambda f
\end{gathered}
$$

Where the terms $P$ and $v$ are the acoustic pressure of sound in specific medium in the unit( $(\mathrm{Pa})$ and the speed of sound in specific medium in the unit $\left(\mathrm{m} / \mathrm{s}^{2}\right), \omega$ is the angular frequencyin the unit $(\mathrm{Hz}), \lambda$ is the wave length in uit $(\mathrm{m})$ and $f$ is the frequencyin the unit $(\mathrm{Hz})$. Although there are several studies $[25,26,27]$ on sound absorption coefficient both theoretically and experimentally, the models which show the relationship between thickness, porosity, weight of fabric and frequency of the value of sound absorption coefficient have not been formulated in more detail about these variables. In this study, the sound absorption coefficient equation was examined using the modification of the sound wave equation and using genetic algorithms with computation to get a better model. This new model included the influence of sound absorption coefficient phenomenon caused by porosity, the weight of the fabric, the thickness of fabric as well as the frequency of the sound wave.

\section{Materials and methods}

\section{1. Modeling of Sound Absorption Coefficient on Fabric}

Suppose two acoustic media 2 and 1 interfacing through a plane surface and characterized by the surface impendence $Z_{2}$ and $Z_{1}$ (Figure 1). The acoustic wave moves from medium 1 to medium 2 . The sound wave moves
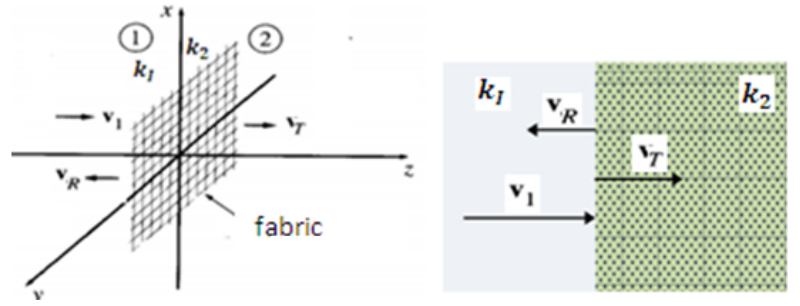

Figure 1. An acoustic wave moves from medium 1 to medium 2

from medium 1 is partly reflected and partly refracted (transmitted) as well as partly absorbed.

If in the case the medium 1 is the air, its surface impedance can be written as Eq. (14)

$$
Z=\rho_{o} c_{o}
$$

Where the terms $\rho_{o}$ and $c_{o}$ are the density of the ambient air in the unit $\left(\mathrm{kg} / \mathrm{m}^{3}\right)$ and the speed of sound in the ambient air in the unit $\left(\mathrm{m} / \mathrm{s}^{2}\right)$. The continuity equation of sound wave propagation can be formulated as follows Eq. (15)

$$
\nabla . \rho v=-\frac{\partial \rho}{\partial t}
$$

Where the terms $\rho$ and $v$ are the density of sound in specific medium in the unit $\left(\mathrm{kg} / \mathrm{m}^{3}\right)$ and the speed of sound in specific medium in the unit $\left(\mathrm{m} / \mathrm{s}^{2}\right)$. With the Cauchy equation, it can be formulated as follows Eq. (16) and Eq. (17)

$$
\begin{aligned}
& \sigma_{, j}^{i j}+f^{i}=\rho a^{i} \\
& \nabla \cdot \overleftrightarrow{\boldsymbol{\sigma}}+\overline{\boldsymbol{f}}=\rho \bar{a}
\end{aligned}
$$

Where $\rho$ is the volume mass density, $\stackrel{\leftrightarrow}{\sigma}$, is a tensor stress which has a general form

$$
\sigma^{i j}=\delta^{i j} \lambda e+2 \mu e^{i j}=\lambda \operatorname{tr}(\overleftrightarrow{e}) \boldsymbol{I}+2 \mu \overleftrightarrow{e}
$$

Where the terms $\sigma^{i j}$ and $e^{i j}$ are the stress tensor and the strain tensor. $\lambda$ and $\mu$ are the elasticity constant. If the magnitude of the gravitational force can be ignored, then by defining that $\nabla \cdot \stackrel{\leftrightarrow}{\sigma}=\bar{\nabla}(-P)$ and the sound wave moves in the $z$ direction, then the wave motion equation can be written as in Eq. (19) and Eq. (20)

$$
\begin{gathered}
\nabla \cdot \overleftrightarrow{\boldsymbol{\sigma}}+\overline{\boldsymbol{f}}=\rho \bar{a} \\
\nabla \cdot \stackrel{\leftrightarrow}{\sigma}+\rho \mathbf{g}+\overline{\boldsymbol{f}}_{\text {ext }}=\rho a
\end{gathered}
$$

Where the terms $\rho$ and $g$ are the density of sound in specific medium in the unit $\left(\mathrm{kg} / \mathrm{m}^{3}\right)$ and the constant of 
gravity in the unit $\left(\mathrm{m} / \mathrm{s}^{2}\right)$. If there is no external force $\bar{f}_{\text {ext }}$ (can be ignored), then it can be written as in Eq. (21) and Eq. (22)

$$
\begin{aligned}
& \bar{\nabla}(-P)=\rho \frac{d v}{d t} \\
& \bar{\nabla}(P)=-\rho \frac{d v}{d t}
\end{aligned}
$$

Divergent of the two segments, we get Eq. (23) to Eq. (25)

$$
\begin{gathered}
\bar{\nabla} \cdot[\bar{\nabla}(P)]=\bar{\nabla} \cdot\left[-\rho \frac{d v}{d t}\right]=\left[-\rho \frac{d(\bar{\nabla} \cdot v)}{d t}\right] \\
\nabla^{2} P=-\frac{d}{d t}(\nabla \cdot \rho v) \\
\nabla^{2} P=\frac{d}{d t}\left(\frac{\partial \rho}{\partial t}\right)
\end{gathered}
$$

Adiabatic expansion process is a process in which there is no change in $Q$ heat in the system on the environment. In the case of adiabatic can be formulated as follows Eq. (26) and Eq. (27)

$$
\begin{gathered}
C d T=-P d V \\
N c_{V} d T=-P d V
\end{gathered}
$$

Where $C$ is the total heat capacity, $C=N c_{v}$ and pressure, $P=\frac{k_{B} T}{V}$ [30], Where the terms $k_{B}, T$ and $V$ are Thermodynamic const, temperature in the unit $(K)$ and volume of matter in the unit $\left(\mathrm{m}^{3}\right)$, hence we get Eq. (28) to Eq. (30)

$$
\begin{gathered}
c_{v} d T=-\frac{k_{B} T d V}{V} \\
\int \frac{d T}{T}=-\frac{k_{B}}{c_{V}} \int \frac{d V}{V} \\
\frac{c_{V}}{k_{B}} \int \frac{d T}{T}=-\int \frac{d V}{V} \\
\frac{c_{V}}{k_{B}} \ln \left(\frac{T}{T_{o}}\right)=\ln \left(\frac{V_{o}}{V}\right)
\end{gathered}
$$

To simplify the calculations, suppose that the ratio $\frac{c_{v}}{k_{B}}=\frac{3}{2}$ [30], then it can be described as follows Eq. (31) and Eq. (32)

$$
\frac{3}{2} \ln \left(\frac{T}{T_{o}}\right)=\ln \left(\frac{V_{o}}{V}\right)
$$

$$
\left(\frac{T}{T_{o}}\right)^{3 / 2}=\frac{V_{o}}{V}
$$

To determine the form of $\mathrm{P}-\mathrm{V}$, it can be described as follows Eq. (33) and Eq. (34)

$$
\begin{gathered}
\left(\frac{P V}{P_{o} V_{o}}\right)^{3 / 2}=\frac{V_{o}}{V} \\
\left(\frac{P}{P_{o}}\right)^{3 / 2}=\left(\frac{V_{o}}{V}\right)^{1+\frac{3}{2}}
\end{gathered}
$$

Where $P=\frac{N k_{B} T}{V}=\rho k_{B} T$, then the relationship between $\rho$ and $V$ is inversely proportional, so we get the following equation (Eq. (35) to Eq. (38))

$$
\begin{gathered}
\left(\frac{P}{P_{o}}\right)^{3 / 2}=\left(\frac{\rho}{\rho_{o}}\right)^{1+\frac{3}{2}} \\
\frac{\rho}{\rho_{o}}=\left(\frac{P}{P_{o}}\right)^{3 / 5} \\
\frac{\rho}{\rho_{o}}=\left(\frac{P}{P_{o}}\right)^{y} \\
\rho=\rho_{o}\left(\frac{P}{P_{o}}\right)^{y}
\end{gathered}
$$

Where $y=\frac{1}{1+\left(\frac{k_{B}}{c_{v}}\right)}=\frac{c_{v}}{c_{v}+k_{B}}$. Suppose that Eq. (38) is differentiated twice with time, then it is obtained Eq. (39) and Eq. (40)

$$
\begin{aligned}
& \frac{1}{\rho_{o}} \frac{\partial^{2} \rho}{\partial t^{2}}=\left(\frac{1}{P_{o}}\right)^{y} y(y-1) P^{y-2}\left(\frac{\partial P}{\partial t}\right)^{2} \\
&+\left(\frac{1}{P_{o}}\right)^{y} y P^{y-1} \frac{\partial^{2} P}{\partial t^{2}} \\
& \frac{\partial^{2} \rho}{\partial t^{2}}= \rho_{o}\left[\left(\frac{1}{P_{o}}\right)^{y} y(y-1) P^{y-2}\left(\frac{\partial P}{\partial t}\right)^{2}\right. \\
&\left.+\left(\frac{1}{P_{o}}\right)^{y} y P^{y-1} \frac{\partial^{2} P}{\partial t^{2}}\right]
\end{aligned}
$$

Substitute Eq. (40) to Eq. (25) then we get Eq. (41)

$$
\begin{aligned}
\nabla^{2} P & =\rho_{o}\left[\left(\frac{1}{P_{o}}\right)^{y} y(y-1) P^{y-2}\left(\frac{\partial P}{\partial t}\right)^{2}\right. \\
& \left.+\left(\frac{1}{P_{o}}\right)^{y} y P^{y-1} \frac{\partial^{2} P}{\partial t^{2}}\right]
\end{aligned}
$$

Suppose that $\left(\frac{\partial P}{\partial t}\right)^{2}$ is very small (there is no sound absorption), then Eq. (42) to Eq. (44)

$$
\nabla^{2} P=\rho_{o}\left[\left(\frac{1}{P_{o}}\right)^{y} y P^{y-1} \frac{\partial^{2} P}{\partial t^{2}}\right]
$$




$$
\begin{aligned}
& \nabla^{2} P=\frac{\rho_{o}}{P_{o}}\left[\left(\frac{P}{P_{o}}\right)^{y-1} y \frac{\partial^{2} P}{\partial t^{2}}\right] \\
& \nabla^{2} P=\frac{\rho_{o}}{P_{o}}\left[\left(\frac{T}{T_{o}}\right)^{y-1} y \frac{\partial^{2} P}{\partial t^{2}}\right]
\end{aligned}
$$

Suppose that $\frac{T}{T_{o}} \approx 1$ (there is no significant temperature difference), then we get Eq. (45)

$$
\nabla^{2} P=\frac{\rho_{o}}{P_{o}}\left[y \frac{\partial^{2} P}{\partial t^{2}}\right]=\frac{1}{v^{2}} \frac{d^{2} P}{d t^{2}}
$$

By remembering that $P=\frac{N k T}{V}=\rho k T$, hence $\frac{P}{\rho}=k_{B} T$ we get Eq. (46) and Eq. (47)

$$
\begin{gathered}
\nabla^{2} P=\frac{1}{k_{B} T_{o}}\left[y \frac{\partial^{2} P}{\partial t^{2}}\right] \\
\nabla^{2} P-\frac{y}{k_{B} T_{o}} \frac{\partial^{2} P}{\partial t^{2}}=\nabla^{2} P-\frac{1}{v^{2}} \frac{d^{2} P}{d t^{2}}=0
\end{gathered}
$$

Then the sound wave velocity is obtained as in Eq. (48)

$$
v=\sqrt{\frac{k_{B} T_{o}}{y}}=\frac{1}{\sqrt{\rho_{o} \epsilon}}=\sqrt{\frac{P_{o}}{\rho_{o} y}}
$$

With the solution of the wave equation is as follows Eq. (49) to Eq. (52)

$$
\begin{gathered}
\frac{1}{v^{2}} \frac{\partial^{2} P(z, t)}{\partial t^{2}}=\nabla^{2} P(z, t) \\
\frac{1}{v^{2}} \frac{1}{P(t)} \frac{\partial^{2} P(t)}{\partial t^{2}}=\frac{1}{P(z)} \frac{\partial^{2} P(z)}{\partial z^{2}}=-k^{2} \\
\frac{1}{v^{2}} \frac{1}{P(t)} \frac{\partial^{2} P(t)}{\partial t^{2}}=-k^{2} \\
\frac{1}{P(t)} \frac{\partial^{2} P(t)}{\partial t^{2}}=-k^{2} v^{2}=-\omega^{2}
\end{gathered}
$$

With the wave number can be written as

$$
k^{2}=\frac{\omega^{2}}{v^{2}}
$$

The $k$ value can be also called the propagation constant (wave propagation) and $\omega$ is the angular frequency of the wave with $v$ called the velocity phase of the wave propagation. The sound pressure value $P(z, t)$ can be described as follows Eq. (53) to Eq. (57):

$$
\frac{\partial^{2} P(z, t)}{\partial z^{2}}=\frac{1}{v^{2}} \frac{d^{2} P(z, t)}{d t^{2}}
$$

$$
\begin{gathered}
P(t) \frac{\partial^{2} P(z)}{\partial z^{2}}=\frac{1}{v^{2}} P(z) \frac{d^{2} P(z)}{d t^{2}} \\
\frac{1}{P(z)} \frac{\partial^{2} P(z)}{\partial z^{2}}=\frac{1}{P(t)} \frac{1}{v^{2}} \frac{d^{2} P(z)}{d t^{2}} \\
\frac{\partial^{2} P(z)}{\partial z^{2}}=-k^{2} P(z) \\
P(z)=P_{o} \cos (k z)
\end{gathered}
$$

Do the same for the function variable $t$, then after a little mathematical calculation, so we get Eq. (58)

$$
\frac{1}{P(t)} \frac{\partial^{2} P(t)}{\partial t^{2}}=-\omega^{2}
$$

Then obtained $P(t)=P_{o} \cos (-\omega t)$, so we get the general function of the wave equation as follows Eq. (59) and Eq. (60)

$$
P(x, t)=P_{o} \cos (k z-\omega t) .
$$

$$
\begin{aligned}
P(z, t) & =P_{o} \cos \left(\frac{2 \pi}{\lambda} z-\frac{2 \pi}{T} t\right) \\
& =P_{o} \cos \left(2 \pi\left(\frac{z}{\lambda}-\frac{t}{T}\right)\right) \\
& =P_{o} \cos \left(\frac{2 \pi}{\lambda}\left(z-\frac{\lambda t}{T}\right)\right) \\
& =P_{o} \cos \left(\frac{2 \pi}{\lambda}(z-v t)\right)
\end{aligned}
$$

For the case $\frac{\partial P}{\partial t}$ is not ignored (there is an absorption of sound waves in medium), then obtained Eq. (61) and Eq. (62)

$$
\begin{aligned}
\nabla^{2} P & =\rho_{o}\left[\left(\frac{1}{P_{o}}\right)^{y} y(y-1) P^{y-2}\left(\frac{\partial P}{\partial t}\right)^{2}\right. \\
& \left.+\left(\frac{1}{P_{o}}\right)^{y} y P^{y-1} \frac{\partial^{2} P}{\partial t^{2}}\right]
\end{aligned}
$$

$$
\begin{aligned}
\nabla^{2} P & =\rho_{o}\left[\frac{1}{P_{o}^{2}}\left(\frac{P}{P_{o}}\right)^{y-2} y(y-1)\left(\frac{\partial P}{\partial t}\right)^{2}\right. \\
& \left.+\left(\frac{P}{P_{o}}\right)^{y-1} \frac{y}{P_{o}} \frac{\partial^{2} P}{\partial t^{2}}\right]
\end{aligned}
$$

If the ratio $\frac{P}{P_{o}}=\frac{T}{T_{o}} \approx 1$, we get Eq. (63) and Eq. (64)

$$
\nabla^{2} P=\left[\frac{\rho_{o}}{P_{o}} y \frac{1}{P_{o}}(y-1)\left(\frac{\partial P}{\partial t}\right)^{2}+\frac{\rho_{o} y}{P_{o}} \frac{\partial^{2} P}{\partial t^{2}}\right]
$$




$$
\begin{aligned}
\nabla^{2} P & =\left[\frac{y(y-1)}{k_{B} T_{o} P_{o}}\left(\frac{\partial P}{\partial t}\right)^{2}+\frac{y}{k_{B} T_{o}} \frac{\partial^{2} P}{\partial t^{2}}\right] \\
& \approx\left[\frac{y(y-1)}{k_{B} T_{o}} \frac{1}{P_{o}}\left(\frac{\partial P}{\partial t}\right)^{2}+\frac{y}{k_{B} T_{o}} \frac{\partial^{2} P}{\partial t^{2}}\right]
\end{aligned}
$$

For case $\frac{P_{o}}{\rho_{o}}=k_{B} T_{o}$ and $\frac{y(y-1)}{P_{o}^{2}}=\sigma$, we get Eq. (65) to Eq. (69)

$$
\begin{gathered}
\nabla^{2} \mathbf{P}-\frac{\rho_{o} y(y-1)}{P_{o}{ }^{2}}\left(\frac{\partial \boldsymbol{P}}{\partial t}\right)^{2}-\frac{\rho_{o} y}{P_{o}} \frac{\partial^{2} \boldsymbol{P}}{\partial t^{2}}=0 \\
\nabla^{2} \mathbf{P}-\rho_{o} \sigma\left(\frac{\partial \boldsymbol{P}}{\partial t}\right)^{2}-\rho_{o} \frac{y}{P_{o}} \frac{\partial^{2} \boldsymbol{P}}{\partial t^{2}}=0 \\
\nabla^{2} \mathbf{P}-\rho_{o} \sigma\left(\frac{\partial \boldsymbol{P}}{\partial t}\right)^{2}-\rho_{o} \epsilon \frac{\partial^{2} \boldsymbol{P}}{\partial t^{2}} \\
\approx \nabla^{2} \mathbf{P}-\rho_{o} \sigma\left(1+\left(\frac{\partial \boldsymbol{P}}{\partial t}-1\right)\right)^{2}-\rho_{o} \epsilon \frac{\partial^{2} \boldsymbol{P}}{\partial t^{2}} \\
=\nabla^{2} \mathbf{P}-\rho_{o} \sigma\left(1+2\left(\frac{\partial \boldsymbol{P}}{\partial t}-1\right)\right)-\rho_{o} \epsilon \frac{\partial^{2} \boldsymbol{P}}{\partial t^{2}}=0 \\
\nabla^{2} \mathbf{P}-\rho_{o} \sigma\left(1+2\left(\frac{\partial \boldsymbol{P}}{\partial t}\right)\right)-\rho_{o} \epsilon \frac{\partial^{2} \boldsymbol{P}}{\partial t^{2}}=\rho_{o} \sigma \\
\nabla^{2} \mathbf{P}-2 \rho_{o} \sigma \frac{\partial \boldsymbol{P}}{\partial t}-\rho_{o} \epsilon \frac{\partial^{2} \boldsymbol{P}}{\partial t^{2}}=\rho_{o} \sigma
\end{gathered}
$$

For case $P_{o}$ is very highand $\rho_{o}$ is very small, then $\sigma=$ $\frac{y(y-1)}{P_{o}^{2}} \approx 0$ with the condition that $\sigma \leq 0$, we get Eq. 70

$$
\begin{aligned}
& \nabla^{2} \mathbf{P}-2 \rho_{o} \sigma \frac{\partial \boldsymbol{P}}{\partial t}-\rho_{o} \epsilon \frac{\partial^{2} \boldsymbol{P}}{\partial t^{2}} \\
& =\nabla^{2} \mathbf{P}-2 \rho_{o} \sigma \frac{\partial \boldsymbol{P}}{\partial t}-\frac{1}{v^{2}} \frac{\partial^{2} \boldsymbol{P}}{\partial t^{2}} \\
& \approx 0
\end{aligned}
$$

For example the solution is $\mathbf{P}=P_{o} e^{i(k z-\omega t)}$, then substitute to the wave equation $\nabla^{2} \mathbf{P}-2 \rho_{o} \sigma \frac{\partial \boldsymbol{P}}{\partial t}-\rho_{o} \epsilon \frac{\partial^{2} \boldsymbol{P}}{\partial t^{2}}=0$, so we get Eq. (71) and Eq. (72)

$$
\begin{gathered}
-k^{2}-2 i \omega \rho_{o} \sigma+\omega^{2} \rho_{o} \epsilon=0 \\
k^{2}=\omega^{2} \rho_{o} \epsilon-2 i \omega \rho_{o} \sigma
\end{gathered}
$$

Suppose that $\sigma^{\star}=2 \sigma$, we get Eq. (73) and Eq. (74)

$$
\begin{aligned}
k^{2} & =\omega^{2} \rho_{o} \epsilon+2 i \omega \rho_{o} \sigma=\omega^{2} \rho_{o} \epsilon\left(1-2 \frac{i \sigma}{\omega \epsilon}\right) \\
& =\omega^{2} \rho_{o} \epsilon\left(1-\frac{i \sigma^{\star}}{\omega \epsilon}\right)
\end{aligned}
$$

where wavenumber can be written as in Eq. (74)

$$
k=\omega \sqrt{\rho_{o} \epsilon}\left(1-\frac{i \sigma^{\star}}{\omega \epsilon}\right)^{1 / 2}
$$

Suppose that $=\alpha-i \beta=\omega \sqrt{\rho_{o} \epsilon}\left(1-\frac{i \sigma^{*}}{\omega \epsilon}\right)^{\frac{1}{2}}$, we get Eq. (75)

$$
\begin{aligned}
k & =\frac{\omega}{v}\left(1-\frac{i \sigma^{\star}}{\omega \epsilon}\right)^{1 / 2}=\frac{\omega}{v}\left(1-\frac{i \sigma^{\star}}{2 \pi f \epsilon}\right)^{1 / 2} \\
& =\frac{\omega}{v}\left(1-i .0,16 \frac{\sigma^{\star}}{f \epsilon}\right)^{1 / 2}
\end{aligned}
$$

Therefore we get Eq. (76)

$$
\begin{aligned}
k^{2} & =(\alpha-i \beta)(\alpha-i \beta)=\alpha^{2}-\beta^{2}-2 i \alpha \beta \\
& =\omega^{2} \rho_{o} \epsilon-i \omega \rho_{o} \sigma^{\star}
\end{aligned}
$$

where $\alpha^{2}-\beta^{2}=\omega^{2} \rho_{o} \epsilon$ and $-2 i \alpha \beta=-i \omega \rho_{o} \sigma^{\star}$ where is $\alpha \beta=\frac{\omega \rho_{o} \sigma^{*}}{2}$. The solution of the sound pressure can be obtained as in Eq. (77)

$$
\begin{aligned}
\boldsymbol{P} & =\boldsymbol{P}_{o} e^{i(k z-\omega t)}=\boldsymbol{P}_{o} e^{i((\alpha-i \beta) z-\omega t)}=\boldsymbol{P}_{o} e^{((i \alpha-\beta) z-i \omega t)} \\
& =\boldsymbol{P}_{o} e^{-\beta z} e^{i(\alpha z-\omega t)}
\end{aligned}
$$

For a case $\alpha=\beta$, then $\beta=\sqrt{\frac{\omega \rho_{\rho} \sigma^{\star}}{2}}$

$$
\beta=\frac{\omega \rho_{o} \sigma^{\star}}{2 \alpha}=\frac{v \rho_{o} \sigma^{\star}}{2}
$$

For the case for $\alpha=\beta$, then with further mathematical calculations in Eq. (76), Eq. (78b) is obtained.

$$
\begin{aligned}
& k=\alpha-i \beta=\omega \sqrt{\frac{\rho_{o} \epsilon}{2}}\left(\sqrt{1+\left(\frac{1}{Q}\right)^{2}}+1\right)^{1 / 2} \\
& -i \omega \sqrt{\frac{\rho_{o} \epsilon}{2}}\left(\sqrt{1+\left(\frac{1}{Q}\right)^{2}}-1\right)^{1 / 2}
\end{aligned}
$$

where $Q=\frac{\omega \epsilon}{\sigma^{\star}}$. If it is defined that the thickness of the absorption $\delta=\frac{1}{\beta}=\frac{2}{v \rho_{o} \sigma^{*}}$ which is the thickness when the sound waves disappear, then for $\omega=\frac{2 \pi}{T}$ and $v=\frac{\lambda}{T}$, we get Eq. (79)

$$
\begin{aligned}
\delta & =\frac{1}{\beta}=\frac{2}{v \rho_{o} \sigma^{\star}}=\frac{2}{\frac{\lambda}{T} \rho_{o}(2 \sigma)}=\frac{2(2 \pi)}{\lambda \omega \rho_{o}(2 \sigma)}=\frac{2 \pi}{\lambda \omega \rho_{o} \sigma} \\
& =\frac{1}{\lambda f \rho_{o} \sigma}
\end{aligned}
$$

the impedance can be written as $Z=\rho v=\rho \frac{\omega}{k}$. By knowing the wavenumber, $k$, then the impedance value $Z$. Large $\beta$ can be determined related to the energy dissipation (loss of energy) in the sound wave into the form 
of heat energy. Large impedance values show that in the real part there is an acoustic resistance, while in the imaginary part there is an acoustic reactant or a phase change or energy storage mechanism. The value of reflection and transmission without any absorption event can be described as follows for incident wave pressure, reflection pressure and transmittance pressure as shown in Eq. (80) to Eq. (82)

$$
\begin{gathered}
\boldsymbol{P}_{\boldsymbol{i}}(z, t)=P_{i} \cos \left(k_{1} z-\omega t\right) \\
\boldsymbol{P}_{\boldsymbol{R}}(z, t)=P_{R} \cos \left(-k_{1} z-\omega t\right) \\
\boldsymbol{P}_{\boldsymbol{T}}(z, t)=P_{T} \cos \left(k_{2} z-\omega t\right)
\end{gathered}
$$

To get the $P_{R}$ and $P_{T}$ values, it can be determined from the boundary conditions, which are as follows, for when $z$ $=0$, we get Eq. (83) to Eq. (85)

$$
\begin{gathered}
\boldsymbol{P}_{\boldsymbol{i}}(0, t)+\boldsymbol{P}_{\boldsymbol{R}}(0, t)=\boldsymbol{P}_{\boldsymbol{T}}(0, t) \\
P_{i} \cos \left(k_{1} 0-\omega t\right)+P_{R} \cos \left(-k_{1} 0-\omega t\right) \\
=P_{T} \cos \left(k_{2} 0-\omega t\right) \\
P_{i}+P_{R}=P_{T}
\end{gathered}
$$

differentiated with $\mathrm{z}$, then Eq. (83) can be written as shown in Eq. (86) to Eq. (88)

$$
\begin{gathered}
\frac{\partial}{\partial z}\left(\boldsymbol{P}_{\boldsymbol{i}}(0, t)+\boldsymbol{P}_{\boldsymbol{R}}(0, t)\right)=\frac{\partial \boldsymbol{P}_{\boldsymbol{T}}(0, t)}{\partial z} \\
k_{1} P_{i}-k_{1} P_{R}=k_{2} P_{T} \\
P_{i}-P_{R}=\frac{k_{2}}{k_{1}} P_{T}
\end{gathered}
$$

Can be eliminated $P_{i}+P_{R}=P_{T}$ and $P_{i}-P_{R}=\frac{k_{2}}{k_{1}} P_{T}$, we get Eq. (89)

$$
P_{T}=\frac{2 k_{1}}{k_{1}+k_{2}} P_{i}
$$

With $v=\frac{\omega}{k}=\frac{z}{\rho}$, for a medium that has more pivot, it can be assumed that $\rho_{1} \approx \rho_{2}$, then we get Eq. (90) and Eq. (91)

$$
\begin{aligned}
& P_{T}=\frac{2 k_{1}}{k_{1}+k_{2}} P_{i} \\
& P_{T}=\frac{2 v_{2}}{v_{1}+v_{2}} P_{i}
\end{aligned}
$$

Due to $Z=\rho v$, we get Eq. (92)

$$
P_{T}=\frac{2 Z_{2}}{Z_{1}+Z_{2}} P_{i}
$$

do the same calculation to find the value of $P_{R}$, then Eq. (93) to Eq. (94) are obtained

$$
\begin{aligned}
& P_{R}=\frac{v_{2}-v_{1}}{v_{2}+v_{1}} P_{I} \\
& P_{R}=\frac{Z_{2}-Z_{1}}{Z_{2}+Z_{1}} P_{I}
\end{aligned}
$$

It is defined that the wave intensity is as in Eq. (95) [25]

$$
I=\frac{P_{\text {power }}}{A}\left(\frac{\mathrm{kg}}{\mathrm{s}^{3}}\right)
$$

or it can be written as shown in Eq. (96) [25]

$$
I=\frac{P_{\text {pressure }}{ }^{2}}{Z}\left(\frac{\mathrm{kg}}{\mathrm{s}^{3}}\right)
$$

It is defined that the reflection coefficient, transmission coefficient and absorption coefficient are as follows as Eq. (96)

$$
a_{R}=\frac{I_{R}}{I_{i}}=\frac{\frac{P_{R}{ }^{2}}{Z_{1}}}{\frac{P_{i}{ }^{2}}{Z_{1}}}=\frac{P_{R}{ }^{2}}{P_{i}{ }^{2}}=\left(\frac{Z_{2}-Z_{1}}{Z_{2}+Z_{1}}\right)^{2}=R^{2}
$$

if $Z_{1}$ is the impedance in ambient air, then $Z_{1}=\rho_{o} v$, we get Eq. (98)

$$
a_{R}=\left(\frac{\frac{Z_{2}}{Z_{1}}-1}{\frac{Z_{2}}{Z_{1}}+1}\right)^{2}=\left(\frac{\frac{Z_{2}}{\rho_{o} V}-1}{\frac{Z_{2}}{\rho_{o} v}+1}\right)^{2}=R^{2}
$$

We get that $\frac{Z_{2}}{\rho_{o} V}=\frac{1+R}{1-R}$ and also we can found Eq. (99)

$$
\begin{aligned}
a_{T} & =\frac{I_{T}}{I_{i}}=\frac{\frac{P_{T}{ }^{2}}{Z_{2}}}{\frac{P_{i}^{2}}{Z_{1}}}=\frac{P_{T}^{2}}{P_{i}^{2}} \frac{Z_{1}}{Z_{2}}=\left(\frac{2 Z_{2}}{Z_{1}+Z_{2}}\right)^{2} \frac{Z_{1}}{Z_{2}} \\
& =\frac{4 Z_{2} Z_{1}}{\left(Z_{1}+Z_{2}\right)^{2}}=T^{2}
\end{aligned}
$$

$a_{T}$ is called the reflection factor or the pressure reflection coefficient. Sound absorption coefficient can be defined as Eq. (100)

$$
a_{T} a_{a}=\frac{I_{a}}{I_{i}}=\frac{P_{A}^{2}}{P_{i}^{2}} \frac{Z_{1}}{Z_{2}}=A^{2}
$$

In this study, there are two types of equations in the event of the propagation of sound waves in a medium, namely the absorption and the absence of absorption in the medium. in the absence of absorption, it can be written that $a_{R}+a_{T}=1$, while in the event of absorption by the medium, it can be written $a_{R}+a_{T}+a_{A}=1$, so 
the sound absorption coefficient can be written as follows $a_{a}=1-a_{R}-a_{T}$, then obtained Eq. (101) and Eq. (102)

$$
\begin{aligned}
a_{a}+a_{T}= & 1-a_{R}=1-\frac{I_{i}}{I_{R}}=1-\frac{\frac{P_{i}^{2}}{Z_{1}}}{\frac{P_{R}{ }^{2}}{Z_{1}}}=1-\frac{P_{R}^{2}}{P_{i}^{2}} \\
= & 1-\left(\frac{Z_{2}-Z_{1}}{Z_{2}+Z_{1}}\right)^{2} \\
& a_{a}+a_{T}=\frac{4 Z_{2} Z_{1}}{\left(Z_{1}+Z_{2}\right)^{2}}=\frac{4 Z_{2} Z_{1}}{\left(Z_{1}+Z_{2}\right)^{2}}
\end{aligned}
$$

With $Z=\rho v$, which is the speed on the material has the relationship as written $v=\sqrt{\frac{K}{\rho}}=\sqrt{K \rho}$ with $\mathrm{K}$ is the modulus of elasticity in the unit $\left(\mathrm{N} / \mathrm{m}^{2}\right)$ and $\rho\left(\mathrm{kg} / \mathrm{m}^{3}\right)$ is the volume density. The relationship of the parameters can be written as $v_{\text {Solid }}>v_{\text {liquid }}>v_{\text {gas }}$, then $Z_{\text {Solid }}>$ $Z_{\text {liquid }}>Z_{\text {gas. }}$ if $Z_{2}>Z_{1}$ and $\frac{Z_{1}}{Z_{2}}=n$, then we get Eq. (103)

$$
\begin{aligned}
a_{a}+a_{T} & =\frac{4 Z_{2} Z_{1}}{\left(Z_{1}+Z_{2}\right)^{2}}=\frac{4 Z_{2} Z_{1}}{\left(Z_{1}+Z_{2}\right)^{2}} \\
& =\frac{4 Z_{2} Z_{1}}{\left(Z_{2}\left(1+\frac{Z_{1}}{Z_{2}}\right)\right)^{2}}=\frac{4 Z_{2} Z_{1}}{Z_{1}^{2}\left(1+\frac{Z_{1}}{Z_{2}}\right)^{2}} \\
& =\frac{4 Z_{1}}{Z_{2}\left(1+\frac{Z_{1}}{Z_{2}}\right)^{2}}=\frac{4 n}{(1+n)^{2}}
\end{aligned}
$$

Suppose that $Z_{1}=1$, then $a_{a}$ depends on $Z_{2}$, by remembering that $Z=\rho v$, then we get Eq. (104) to Eq. (106)

$$
\begin{aligned}
a_{a}+a_{T}=\frac{4 Z_{2}}{\left(1+Z_{2}\right)^{2}}=\frac{4 v_{2}}{\left(1+v_{2}\right)^{2}} \\
a_{a}+a_{T}=\frac{4 \omega_{2} / k_{2}}{\left(1+\frac{\omega_{2}}{k_{2}}\right)^{2}}=\frac{42 \pi f_{2} / k_{2}}{\left(1+\frac{2 \pi f_{2}}{k_{2}}\right)^{2}} \\
=\frac{4 f_{2} / J_{n m}}{\left(1+f_{2} / J_{n m}\right)^{2}}=\frac{4 m}{(1+m)^{2}}
\end{aligned}
$$

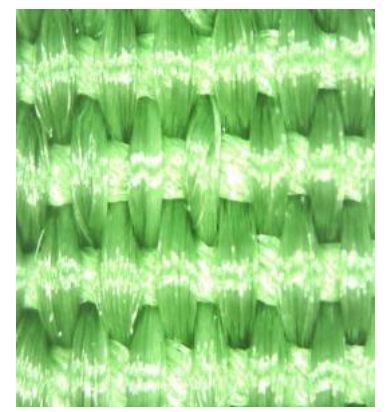

(a)

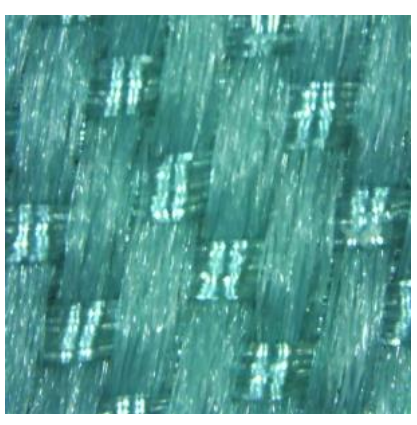

(b)

$$
a_{a}=\frac{4 m}{(1+m)^{2}}-a_{T}
$$

Which requires that the value is $m=\frac{f_{2}}{J_{n m}}$. with $J_{n m}$ is a constant depending on the material, $f_{2}$ is the frequency

\section{Results and Discussion}

In this study, the value of the sound absorption coefficient was related to a constant variable on woven fabric. The types of woven fabric in this study were commercial woven fabrics, such as plain, rips, twill $2 / 1$ and satin fabrics and we used polyester fabrics (purchased in Bandung, Indonesia). The fabric thickness was measured at a pressure of $5 \mathrm{gr} / \mathrm{cm}^{2}$ using a standard compression tester. The fabric density and porosity were measured with the standard tester (Textile Research Center, Bandung, Indonesia). Porosity is defined as the ratio of the void space within the material to its total displacement volume [29]. In this study, The fabric characteristic was shown in Table 1 and Figure 2. The measurement of the sound absorption coefficient of the samples was conducted using the impedance tube (physics laboratory, Bandung Institute of Technology, Indonesia). The device had absorption frequency ranging from $64 \mathrm{~Hz}$ to $6300 \mathrm{~Hz}$. The schematic diagram of the test can be shown in Figure 3

Based on experimental data for plain fabric, the correlation between sound absorption coefficient and frequency values was obtained as shown in Table 2 .

For a case $J_{n m}=1000$ and we evaluate $f_{2}$ in range of $50 \mathrm{~Hz}$ to $2000 \mathrm{~Hz}$ with $a_{T}=0.4$, we get $a_{a}$. By using Eq. (106), then we get Eq. (107)

$$
a_{a}=\frac{4 m}{(1+m)^{2}}-a_{T}=\frac{4\left(\frac{f_{2}}{J_{n m}}\right)}{\left(1+\left(\frac{f_{2}}{J_{n m}}\right)\right)^{2}}-a_{T}
$$

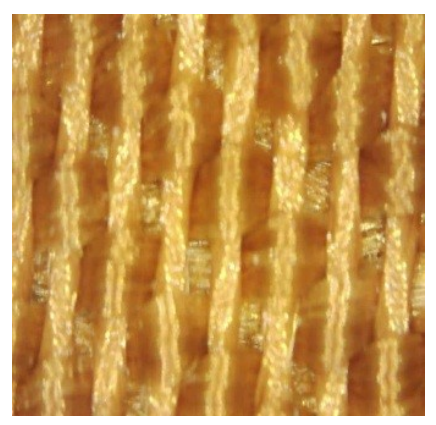

(c)

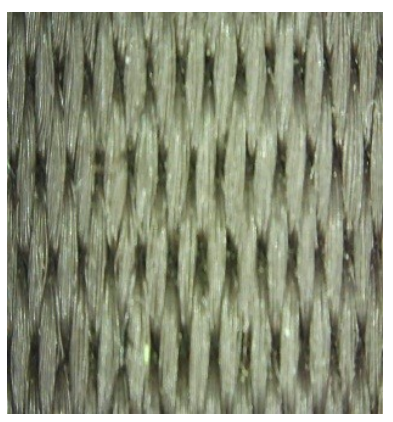

(d)

Figure 2. Type of woven fabric used in this study: a) plain; b)satin; c)twill $2 / 1$;d) rips 


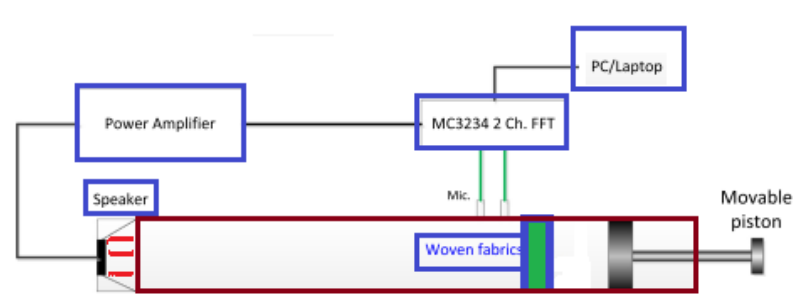

Figure 3. The schematic diagram of the test (Physics Laboratory, Institut Teknologi Bandung (Bandung Institute of Technology))

Using curve fitting and genetic algorithm, then we get Eq. (108)

$$
a_{a}=\frac{4 m}{(1+m)^{2}}-a_{T}=\frac{4\left(\frac{f_{2}}{1000}\right)}{\left(1+\left(\frac{f_{2}}{1000}\right)\right)^{2}}-0.4
$$

The graph results between experiments and models can be shown in Figure 4

Based on experimental data for satin, the correlation between sound absorption coefficient and frequency values was obtained as shown in Table 3 .

For $J_{n m}=650$ and $f_{2}$ analyzed for $50 \mathrm{~Hz}$ to $2000 \mathrm{HZ}$ and $a_{T}=0.65$, it was found that the magnitude of $a_{a}$ is as follows. By using Eq. (106), Eq. (109) is obtained.

$$
a_{a}=\frac{4 m}{(1+m)^{2}}-a_{T}=\frac{4\left(\frac{f_{2}}{J_{n m}}\right)}{\left(1+\left(\frac{f_{2}}{J_{n m}}\right)\right)^{2}}-a_{T}
$$

Table 1. Fabric structural properties (physics evaluation laboratory, Politeknik STTT Bandung and Textile Research Center Bandung, Indonesia)

\begin{tabular}{lccc}
\hline Type of Fabric & $\begin{array}{c}\text { Fabric Weight } \\
\left(\mathrm{g} / \mathrm{m}^{2}\right)\end{array}$ & $\begin{array}{c}\text { Fabric } \\
\text { Thickness } \\
(\mathrm{mm})\end{array}$ & Porosity (\%) \\
\hline Plain & 160 & 0.51 & 0.76 \\
Satin & 148 & 0.59 & 0.82 \\
Twill 2/1 & 154 & 0.53 & 0.79 \\
Rips & 151 & 0.56 & 0.81 \\
\hline
\end{tabular}

Table 2. A sound absorption relationship Coefficient of the frequency of plain fabric

\begin{tabular}{rr}
\hline$a_{\operatorname{aexp}}$ & $f(H z)$ \\
\hline 0.15 & 210 \\
0.425 & 500 \\
0.525 & 710 \\
0.55 & 1000 \\
0.475 & 1210 \\
0.425 & 1500 \\
0.2 & 2000 \\
\hline
\end{tabular}

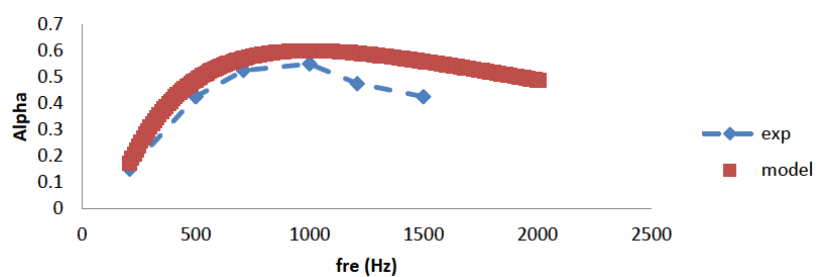

Figure 4. Graphical results between the experiment and the model of the sound absorption coefficient, $a_{a}$, for plain fabric

Table 3. A sound absorption relationship Coefficient of the frequency of Satin fabric

\begin{tabular}{rr}
\hline$a_{a \exp }$ & $f(H z)$ \\
\hline 0.1 & 210 \\
0.223 & 500 \\
0.3 & 710 \\
0.325 & 1000 \\
0.223 & 1210 \\
0.222 & 1500 \\
0.125 & 1710 \\
\hline
\end{tabular}

By using curve fitting and genetic algorithm, we get Eq. (110)

$$
a_{a}=\frac{4 m}{(1+m)^{2}}-a_{T}=\frac{4\left(\frac{f_{2}}{650}\right)}{\left(1+\left(\frac{f_{2}}{650}\right)\right)^{2}}-0.65
$$

The graph results between experiments and models can be shown in Figure 5

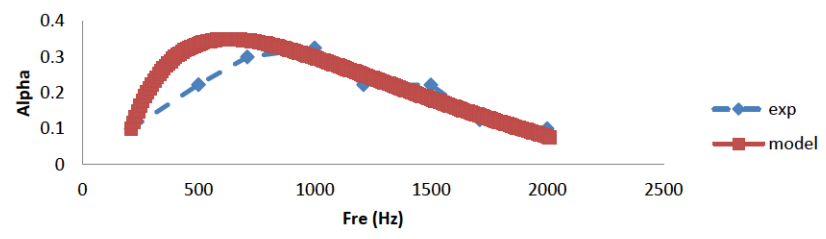

Figure 5. Graphical results between the experiment and the model of the sound absorption coefficient, $a_{a}$, for Satin fabric

Based on experimental data for Rips fabric, the correlation between sound absorption coefficient and frequency values was obtained as shown in Table 4.

For a case $J_{n m}=730$ and we evaluate $f_{2}$ in range of $50 \mathrm{~Hz}$ to $2000 \mathrm{~Hz}$ with $a_{T}=0.6$, we get $a_{a}$. By using Eq. (106), then we get Eq. (111)

$$
a_{a}=\frac{4 m}{(1+m)^{2}}-a_{T}=\frac{4\left(\frac{f_{2}}{J_{n m}}\right)}{\left(1+\left(\frac{f_{2}}{J_{n m}}\right)\right)^{2}}-a_{T}
$$


Table 4. A sound absorption relationship Coefficient of the frequency of Rips fabric

\begin{tabular}{rr}
\hline$a_{a \exp }$ & $f(H z)$ \\
\hline 0.12 & 210 \\
0.26 & 500 \\
0.38 & 710 \\
0.41 & 1000 \\
0.31 & 1210 \\
0.25 & 1500 \\
0.21 & 1710 \\
\hline
\end{tabular}

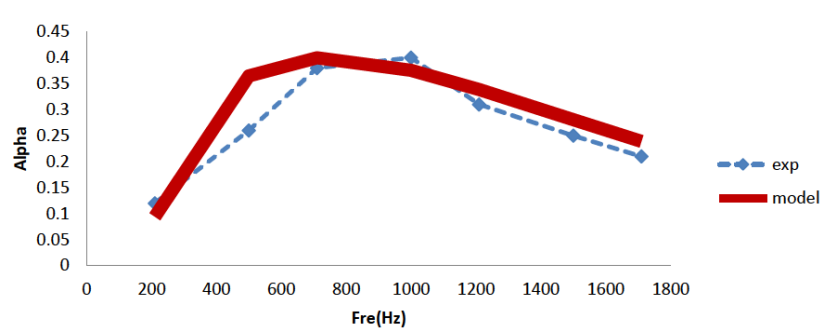

Figure 6. Graphical results between the experiment and the model of the sound absorption coefficient, $a_{a}$, for Rips fabric

Using curve fitting and Genetic Algorithm, then we get Eq. (112)

$$
a_{a}=\frac{4 m}{(1+m)^{2}}-a_{T}=\frac{4\left(\frac{f_{2}}{730}\right)}{\left(1+\left(\frac{f_{2}}{730}\right)\right)^{2}}-0.6
$$

The graph results between experiments and models can be shown in Figure 6

Based on experimental data for Twill 2/1 fabric, the correlation between sound absorption coefficient and frequency values was obtained as shown in Table 5.

Table 5. A sound absorption relationship coefficient of the frequency of Twill 2/1 fabric

\begin{tabular}{rr}
\hline$a_{a \exp }$ & $f(H z)$ \\
\hline 0.14 & 210 \\
0.38 & 500 \\
0.48 & 710 \\
0.45 & 1000 \\
0.4 & 1210 \\
0.33 & 1500 \\
0.22 & 1710 \\
\hline
\end{tabular}

For a case $J_{n m}=760$ and we evaluate $f_{2}$ in range of $50 \mathrm{~Hz}$ to $2000 \mathrm{~Hz}$ with $a_{T}=0.52$, we get $a_{a}$. By using Eq. (106), then we get Eq. (113)

$$
a_{a}=\frac{4 m}{(1+m)^{2}}-a_{T}=\frac{4\left(\frac{f_{2}}{J_{n m}}\right)}{\left(1+\left(\frac{f_{2}}{J_{n m}}\right)\right)^{2}}-a_{T}
$$

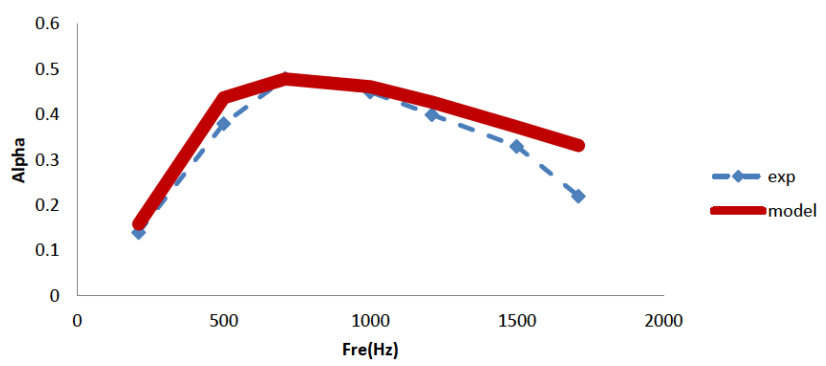

Figure 7. Graphical results between the experiment and the model of the sound absorption coefficient, $a_{a}$, for twill 2/1 fabric

Using curve fitting and Genetic Algorithm, then we get Eq. (114)

$$
a_{a}=\frac{4 m}{(1+m)^{2}}-a_{T}=\frac{4\left(\frac{f_{2}}{760}\right)}{\left(1+\left(\frac{f_{2}}{760}\right)\right)^{2}}-0.52
$$

The graph results between experiments and models can be shown in Figure 7

Based on the calculation results, the plain fabric specifications in the first model follow the following formula Eq. (115)

$$
a_{a}=\frac{4\left(\frac{f_{2}}{1000}\right)}{\left(1+\left(\frac{f_{2}}{1000}\right)\right)^{2}}-0.4
$$

The specifications of the satin fabric in the second model follow the following formula Eq. (116)

$$
a_{a}=\frac{4\left(\frac{f_{2}}{650}\right)}{\left(1+\left(\frac{f_{2}}{650}\right)\right)^{2}}-0.65
$$

The specifications of the Rips fabric in the second model follow the following formula Eq. (117)

$$
a_{a}=\frac{4\left(\frac{f_{2}}{730}\right)}{\left(1+\left(\frac{f_{2}}{730}\right)\right)^{2}}-0.6
$$

The specifications of the Twill2/1 fabric in the second model follow the following formula Eq. (118)

$$
a_{a}=\frac{4\left(\frac{f_{2}}{760}\right)}{\left(1+\left(\frac{f_{2}}{760}\right)\right)^{2}}-0.52
$$

In curve fitting using Genetic Algorithm, there are three constants that was connected with fabric parameters, such as weight, thickness and porosity (Table 4).

To determine the relationship between weight, thickness, porosity, $J_{n m}$ and $a_{T}$, therefore we used in a simple 
Table 6. A parameter of plain fabric and satin fabric

\begin{tabular}{cccccc}
\hline Type of fabric & Weight, $w,\left(\mathrm{~g} / \mathrm{m}^{2}\right)$ & Thickness, $d,(\mathrm{~mm})$ & $\phi$ Porosity $(\%)$ & $J_{n m}$ & $a_{T}$ \\
\hline Plain & 160 & 0.51 & 0.77 & 0.4 & 1000 \\
Satin & 148 & 0.59 & 0.82 & 650 & 0.65 \\
Twill 2/1 & 154 & 0.53 & 0.79 & 760 & 0.52 \\
Rips & 151 & 0.56 & 0.81 & 730 & 0.6 \\
\hline
\end{tabular}

form using the following Eq. (119) to Eq. (122). The value $a_{T}$ had a relationship that is directly proportional to thickness and porosity, where as $J_{n m}$ had a relationship that is directly proportional to weight

$$
\begin{aligned}
& \sum_{i=1}^{n} \hat{y}_{i}=a_{0}+a_{1} \sum x_{i 1}+a_{2} \sum x_{i 2}+\cdots \\
&+a_{k} \sum x_{i k} \\
& \hat{y}_{1}=a_{0}+a_{1} x_{11}+a_{2} x_{12}++a_{3} x_{13}+\cdots+a_{k} x_{1 k} \\
& \hat{y}_{2}= a_{0}+a_{1} x_{21}+a_{2} x_{22}++a_{3} x_{23}+\cdots+a_{k} x_{2 k} \\
& \hat{y}_{n}= a_{o}+a_{1} x_{n 1}+a_{2} x_{n 2}++a_{3} x_{n 3}+\cdots+a_{k} x_{n k} \\
&\left(\begin{array}{c}
\hat{y}_{1} \\
: \\
\hat{y}_{n}
\end{array}\right)=\left(\begin{array}{ccc}
1 & \cdots & x_{1 k} \\
1 & \ddots & : \\
1 & \cdots & x_{n k}
\end{array}\right)\left(\begin{array}{c}
a_{0} \\
: \\
a_{k}
\end{array}\right) \\
& \hat{y}=x_{i k} a_{k} \\
& \hat{y}=X a
\end{aligned}
$$

The difference between experimental data and predictive modeling data is referred to as error $\epsilon$ and has a value of Eq. (123)

$$
\sum_{i=1}^{n}\left(y_{i}-\hat{y}_{i}\right)=\epsilon
$$

If Eq. (123) is squared, it will produce Eq. (124) as shown below

$$
\epsilon^{T} \epsilon=L
$$

To find the value of $a$, optimization can be done through the squared differential Equation (124) with respect to $a$, hence we get Eq. (125) to Eq. (130)

$$
\begin{gathered}
\frac{d L}{d a}=\frac{d}{d a}\left(y^{T} y-y^{T} X a-\left(y^{T} X a\right)^{T}+a^{T} X^{T} X a\right) \\
=-2 y^{T} X+2 a^{T} X^{T} X=0 \\
2 y^{T} X=2 a^{T} X^{T} X
\end{gathered}
$$

$$
\begin{gathered}
a^{T} X^{T} X=y^{T} X \text { with } a^{T}=\left(X^{T} X\right)^{-1} y^{T} X \\
\left(a^{T} X^{T} X\right)^{T}=\left(y^{T} X\right)^{T} \\
\left(X^{T} X\right) a=X^{T} y \\
a=\left(X^{T} X\right)^{-1} X^{T} y \\
\hat{y}=X a=X\left(X^{T} X\right)^{-1} X^{T} y
\end{gathered}
$$

Based on Equation (130) above, with the matrix X, therefore we obtained Eq. (131) to Equation (134) below

$$
\begin{gathered}
a_{T}=a_{o} d^{a_{1}} \phi^{a_{2}} \\
\ln a_{T}=\ln a_{o}+a_{1} \ln (d)+a_{1} \ln (\phi) \\
Y=A_{o}+A_{1} X_{1}+A_{2} X_{2}
\end{gathered}
$$

by using MATLAB programming, we get Eq. (133) with $a_{o}=e^{A o}$ and $a_{1}=A_{1}, a_{2}=A_{2}$, we get $a_{T}=$ $e^{0.3778} d^{-11} \phi^{-42.7}$. Do the same for $J_{n m}$ as a function of weight, then we get $J_{n m}=5.39 \mathrm{w}$

$$
\begin{aligned}
a_{a} & =\frac{4 m}{(1+m)^{2}}-a_{T}=\frac{4\left(\frac{f_{2}}{J_{n m}}\right)}{\left(1+\left(\frac{f_{2}}{J_{n m}}\right)\right)^{2}}-a_{T} \\
& =\frac{4\left(\frac{f_{2}}{5.39 w}\right)}{\left(1+\left(\frac{f_{2}}{5.39 w}\right)\right)^{2}}-\left(e^{0.3778} d^{-11} \phi^{-42.7}\right)
\end{aligned}
$$

Then we get the general formula of sound absorption coefficient as a function of weight, thickness and porosity that it can be formulated using the following Eq. (135)

$$
a_{a}=\frac{4\left(\frac{f_{2}}{C_{1} w}\right)}{\left(1+\left(\frac{f_{2}}{C_{1} w}\right)\right)^{2}}-\left(C_{2} d^{-C_{3}} \phi^{-C_{4}}\right)
$$

$a_{a}$ is the sound absorption coefficient, $\phi(\%)$ is the porosity, $d(\mathrm{~mm})$ is the thickness of fabric, $f(\mathrm{~Hz})$ is the frequency and $w\left(\mathrm{~g} / \mathrm{m}^{2}\right)$ is the weight of fabric. The results 
Table 7. The Comparison between models and literature

\begin{tabular}{|c|c|c|}
\hline $\begin{array}{l}\text { The properties of } \\
\text { sound }\end{array}$ & Model & Literature \\
\hline Wave equation & $\begin{array}{l}\nabla^{2} \mathbf{P}-2 \rho_{o} \sigma \frac{\partial \mathbf{P}}{\partial t}-\rho_{o} \epsilon \frac{\partial^{2} \mathbf{P}}{\partial t^{2}} \approx 0 \\
\nabla^{2} \mathbf{P}-2 \rho_{o} \sigma \frac{\partial \mathbf{P}}{\partial t}-\frac{1}{v^{2}} \frac{\partial^{2} \mathbf{P}}{\partial t^{2}}=0\end{array}$ & $\nabla^{2} P-\frac{1}{v^{2}} \frac{d^{2} P}{d t^{2}}=0[25]$ \\
\hline Sound pressure & $\mathbf{P}=P_{o} e^{-\beta z} e^{i(\alpha z-\omega t)}$ & $\mathbf{P}(z, t)=P_{o} \cos (\mathbf{k} \cdot \mathbf{r}-\omega \mathbf{t})[25]$ \\
\hline Impedance & $\begin{array}{c}Z=\rho v=\mu \frac{\omega}{k}=\frac{\mu \omega}{\alpha+i \beta}=\frac{\mu \omega}{\alpha+i \beta} \frac{\alpha-i \beta}{\alpha-i \beta}=\frac{\mu \omega \alpha-i \mu \omega \beta}{\alpha^{2}-\beta^{2}}= \\
Z_{\text {real }}-J Z_{\text {img }}\end{array}$ & $\begin{array}{c}Z=\rho_{o} c_{o}\left(1+0.0571 X^{-0.754}-j 0.087 X^{-0.732}\right) \\
Z=r+j \omega m=\frac{32 \eta t}{\rho_{o} c_{0} d^{2} p}\left(\sqrt{1+\frac{x^{2}}{32}}+\frac{x d \sqrt{2}}{8 t}\right)+ \\
\quad \frac{j \omega t}{p c_{o}}\left(1+\left(9+\frac{x^{2}}{2}\right)^{-1 / 2}+\frac{0.85 d}{t}\right)[4,28]\end{array}$ \\
\hline Wavenumber & $\begin{array}{c}k=\alpha-i \beta=\omega \sqrt{\frac{\rho_{o} \epsilon}{2}}\left(\sqrt{\left.1+\left(\frac{1}{Q}\right)^{2}+1\right)-}\right. \\
i \omega \sqrt{\frac{\rho_{o} \epsilon}{2}}\left(\sqrt{1+\left(\frac{1}{Q}\right)^{2}}-1\right)^{1 / 2} \\
\text { where } Q=\frac{\omega \epsilon}{\sigma^{*}}\end{array}$ & $k=\omega / c_{o}\left(1+0.0978 X^{-0.700}-j 0.189 X^{-0.595}\right)[25]$ \\
\hline $\begin{array}{l}\text { Sound absorption } \\
\text { coefficient }\end{array}$ & $\begin{array}{c}a_{a}=1-\left(\frac{z_{2}-Z_{1}}{Z_{2}+Z_{1}}\right)^{2}-a_{T} \\
a_{a}=\frac{4 m}{(1+m)^{2}}-a_{T} \\
a_{a}=\frac{4\left(\frac{f_{2}}{c_{1} w}\right)}{\left(1+\left(\frac{f 2}{c_{1} w}\right)\right)^{2}}-\left(C_{2} d^{-C_{3}} \phi^{-C_{4}}\right)\end{array}$ & $\begin{array}{c}a_{a}=\frac{4 \operatorname{Re}\left\{Z_{\text {tot }}\right\}}{\left(1+\operatorname{Re}\left\{Z_{\text {tot }}\right\}\right)^{2}+\left(\operatorname{Img}\left\{Z_{\text {tot }}\right\}\right)^{2}}[26] \\
\alpha=\frac{4 \operatorname{Re}\left\{Z_{\text {tot }}\right\}}{\left(1+\operatorname{Re}\left\{Z_{\text {tot }}\right\}\right)^{2}}=1-\left(\frac{Z_{2}-\rho_{0} c_{0}}{Z_{2}+\rho_{0} c_{0}}\right)^{2}[27]\end{array}$ \\
\hline
\end{tabular}

showed that factors such as porosity, fabric thickness, fabrication, and frequency affect the value of the sound absorption coefficient, according to Eq. (135). Several studies have shown that in the formation of textile woven fabrics as sound absorbent materials, factors such as porosity, fabric thickness, fabric surface density, and frequency affect the sound absorption coefficient on the fabric $[1,12,13,14,17,18,19,23]$. In some studies, the value of the sound absorption coefficient is influenced by several factors, such as the value of the speed of acoustic wave or speed of sound, the acoustic pressure, the angular frequency, the wavenumber, particle speed of the propagation medium which vibrates around the development of sound and also the acoustic impedance $[1,12,13,14,17,18,19,23,25,26,27]$. According to Atalla et al. [28] and Maa [4], the acoustic impedance of the sound absorber consists of real parts and imaginary parts (resistance impedance for real parts and reactance impedance for imaginary parts). In this study, several new equation results are found that are related to factors that affect the sound absorption coefficient. In Table 7. A new model equation was compared with some of the previous researchers' models.

From the results of prediction and also the validation of experiments, we obtained data as follows Table 8 , Table 9, Table 10 and Table 11.
Table 8. Relationship between sound absorption values Coefficient, $\alpha$, on the frequency, $f$, of plain fabric experiment and model

\begin{tabular}{rrr}
\hline$\alpha_{\text {aexp }}$ & $f(H z)$ & $\alpha_{\text {model }}$ \\
\hline 0.15 & 210 & 0.229638 \\
0.425 & 500 & 0.529071 \\
0.525 & 710 & 0.590539 \\
0.55 & 1000 & 0.594592 \\
0.475 & 1210 & 0.571981 \\
0.425 & 1500 & 0.527331 \\
0.2 & 2000 & 0.442283 \\
\hline
\end{tabular}

Table 9. Relationship between sound absorption values Coefficient, $\alpha$, on the frequency, $f$, of satin fabric experiment and model

\begin{tabular}{rrr}
\hline$\alpha_{\text {aexp }}$ & $f(H z)$ & $\alpha_{\text {model }}$ \\
\hline 0.1 & 210 & 0.294277 \\
0.22 & 500 & 0.345752 \\
0.3 & 710 & 0.338852 \\
0.32 & 1000 & 0.310553 \\
0.22 & 1210 & 0.260441 \\
0.21 & 1500 & 0.222064 \\
0.13 & 1710 & 0.170229 \\
\hline
\end{tabular}

The $\mathrm{R}^{2}$ value obtained was 0.8399 with the relationship between the model and the experimental results shown in Figure 8 
Table 10. Relationship between sound absorption values Coefficient, $\alpha$, on the frequency, $f$, of twill $2 / 1$ fabric experiment and model

\begin{tabular}{rrr}
\hline$\alpha_{\text {aexp }}$ & $f(H z)$ & $\alpha_{\text {model }}$ \\
\hline 0.14 & 210 & 0.158499 \\
0.38 & 500 & 0.43742 \\
0.48 & 710 & 0.478843 \\
0.45 & 1000 & 0.461405 \\
0.4 & 1210 & 0.427821 \\
0.33 & 1500 & 0.372787 \\
0.22 & 1710 & 0.332071 \\
\hline
\end{tabular}

Table 11. Relationship between sound absorption values Coefficient, $\alpha$, on the frequency, $f$, of Rips fabric experiments and models

\begin{tabular}{rrr}
\hline$\alpha_{\text {aexp }}$ & $f(H z)$ & $\alpha_{\text {model }}$ \\
\hline 0.12 & 210 & 0.093979 \\
0.26 & 500 & 0.365034 \\
0.38 & 710 & 0.399807 \\
0.4 & 1000 & 0.375642 \\
0.31 & 1210 & 0.338782 \\
0.25 & 1500 & 0.280774 \\
0.21 & 1710 & 0.238686 \\
\hline
\end{tabular}

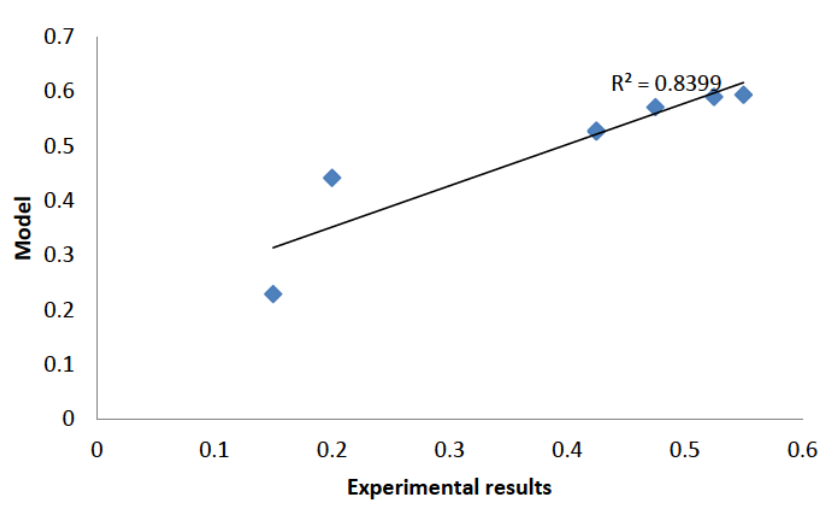

Figure 8. The relationship between the model and the experimental results of plain fabric

The $R^{2}$ value was obtained 0.2815 , with the relationship between the model and the experimental results shown in Figure 9

The $R^{2}$ value obtained was 0.909 , with the relationship between the model and the experimental results shown in Figure 10

The $R^{2}$ value was 0.8303 , with the relationship between the model and the experimental results shown in Figure 11

In this model, the sound absorption coefficient equation was obtained by modeling the sound wave equation and by curve fitting using genetic algorithms. This model included the influence of the sound absorption coefficient

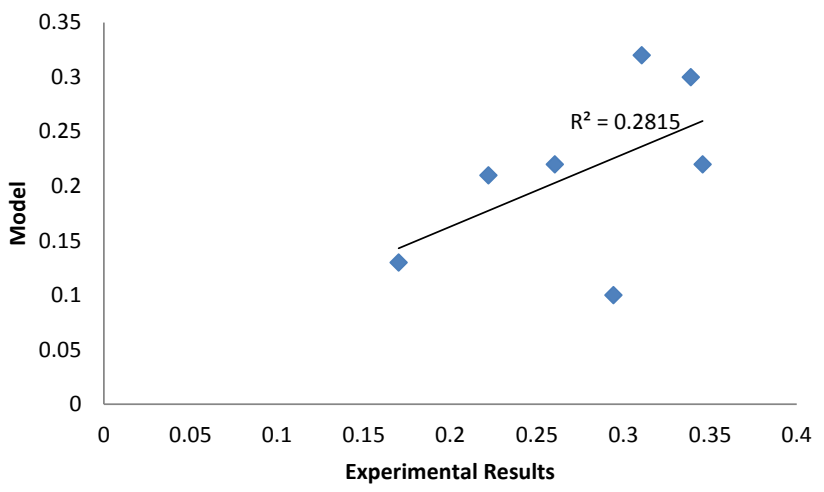

Figure 9. The relationship between the model and the experimental results of satin fabric

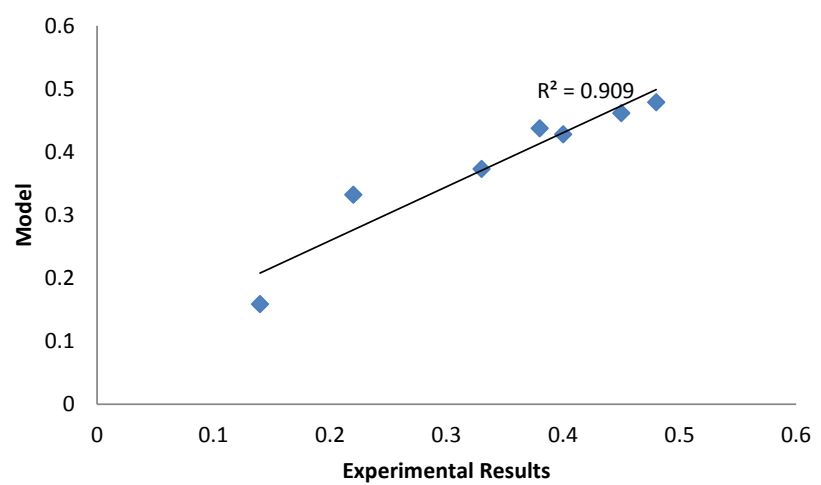

Figure 10. The relationship between the model and the experimental results of twill 2/1 fabric

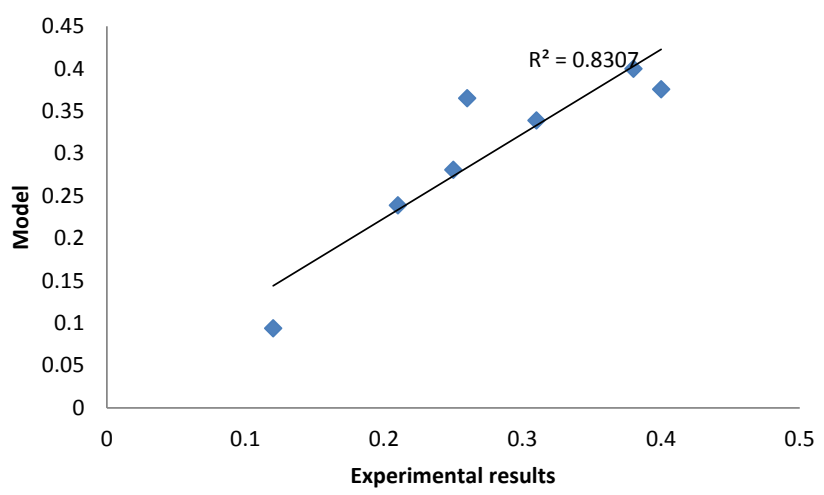

Figure 11. The relationship between the model and the experimental results of rips fabric

phenomenon caused by porosity, the weight of the fabric, the thickness of fabric as well as the frequency of the sound wave. In this study, experimental data showed a good agreement with the model. In this study, the results of the model and experimental validation show quite good prediction and we had got the general formula of sound absorption coefficient as a function of weight, thickness, and porosity. The results showed that for twill 2/1, rips, 
and plain fabrics had good accuracy with an $R^{2}$ value above 0.8 , while for satin, the $R^{2}$ value was 0.2815 . The weakness of this model is that the structural equation of the fabric geometry had not been calculated in detail (using topology concepts and mechanical geometric formulations), but this model provided a good enough analysis to predict the sound absorption coefficient with good results compared to previous methods [25, 26, 27]. In this study, we found that the shape of the structure of woven fabric affects the value of sound absorption coefficient, plain woven fabric had a better fabric structure in increasing the value of sound absorption coefficient compared to other types of woven fabric as said by some researchers [25, 26, 27, 28, 29]. The sound absorption coefficient for plain, satin, rips and twill fabrics was 0.525 , $0.325,0.41$, and 0.48 . We also found that the higher the porosity, the lower is the sound absorption coefficient as reported by some researchers $[25,26,27,28,29]$ and with the porosity values of plain, satin, rips, and twill $2 / 1$ fabrics were $0.77,0.82,0.79$ and 0.81 where the sound absorption coefficient of plain fabric is the largest, while the absorption coefficient of satin is the smallest.

\section{Conclusions}

We have presented a new method for predicting the sound absorption of woven fabrics using modification of sound wave equations and curve fitting using genetic algorithms. A new model for predicting the sound absorption coefficient of woven fabric (plain, twill 2/1, rips and satin fabric) was presented in this article. In this study, the sound absorption coefficient equation was obtained by modeling the sound wave equation and the application of curve fitting using genetic algorithms. This model included the influence of sound absorption coefficient phenomenon caused by porosity, weight of fabric, thickness of fabric as well as frequency of the sound wave. In this study, the results of the model and experimental validation show quite good prediction and we had got the general formula of sound absorption coefficient as a function of weight, thickness, and porosity

Acknowledgment: We would like to express our gratitude to Politeknik STTT Bandung and Universitas Nusa Cendana as the research funders as well as to the contribution of colleagues who helped us during the research and discussion.
Conflict of Interest: All authors declare no conflicts of interest regarding the content and implications of this manuscript

\section{References}

[1] Shoshani, Y., Rosenhouse, G. (1990). Noise absorption by woven fabrics. Applied Acoustics, 30(4), 321-333.

[2] Hanna, Y. I., Kandil, M. M. (1991). Sound absorbing double curtains from local textile materials. Applied Acoustics, 34(4), 281-291.

[3] Park, C.-M., Ih, J. G., Nakayama, Y., Takao, H. (2003). Inverse estimation of the acoustic impedance of a porous woven hose from measured transmission coefficients. Journal of the Acoustical Society of America, 113(1), 128-138.

[4] Maa, D. (1975). Theory and design of microperforated panel sound-absorbing constructions. Scientia Sinica, 18(1), 55-71.

[5] Maa, D. (1985). Microperforated-panel wideband absorbers. Noise Control Engineering Journal, 29, 77-84.

[6] Maa, D. (1998). Potential of microperforated panel absorber. Journal of the Acoustical Society of America, 104, 2861-2866.

[7] Zha, X., Fuchs, H. V., Drotleff, H. (2002). Improving the acoustic working conditions for musicians in small spaces. Applied Acoustics, 63(2), 203-221.

[8] Zhao, X., Fan, X. (2015). Enhancing low frequency sound absorption of micro-perforated panel absorbers by using mechanical impedance plates. Applied Acoustics, 88, 123-128.

[9] Zhao, X. D., Yu, Y. J., Wu, Y. J. (2016). Improving low-frequency sound absorption of microperforated panel absorbers by using mechanical impedance plate combined with Helmholtz resonators. Applied Acoustics, 114, 92-98.

[10] Sakagami, K., Kobatake, S., Kano, K., Morimoto, M., Yairi, M. (2011). Sound absorption characteristics of a single microperforated panel absorber backed by a porous absorbent layer. Acoustics Australia, 39(3), 95-100.

[11] Liu, Z., Zhan, J., Fard, M., Davy, J. L. (2017). Acoustic measurement of a $3 D$ printed microperforated panel combined with a porous material. Measurement: Journal of the International Measurement Confederation, 104, 233-236.

[12] Kang, J., Fuchs, H. V. (1999). Predicting the absorption of open weave textiles and microperforated membranes backed by an air space. Journal of Sound and Vibration, 220(5), 905-920.

[13] Sakagami, K., Morimoto, M., Koike, W. (2006). A numerical study of double-leaf microperforated panel absorbers. Applied Acoustics, 67(7), 609-619.

[14] Sakagami, K., Matsutani, K., Morimoto, M. (2010). Sound absorption of a double-leaf micro-perforated panel with an air-back cavity and a rigid-back wall: detailed analysis with a Helmholtz-Kirchhoff integral formulation. Applied Acoustics, 71(5), 411-417.

[15] Padhye, R., Nayak, R. (2016). Acoustic textiles. Springer (New York).

[16] Pieren, R., Heutschi, K. (2015). Predicting sound absorption coefficients of lightweight multilayer curtains using the equivalent circuit method. Applied Acoustics, 92, 27-41.

[17] Allard, J., Atalla, N. (2009). Propagation of sound in porous media: Modelling soundabsorbing materials. Wiley (New York). 
[18] Ingard, U. (1994). Notes on sound absorption technology. Noise Control Foundation (London).

[19] Bies, D. A., Hansen, C. H. (1980). Flow resistance information for acoustical design. Applied Acoustics, 13(5), 357-391.

[20] Aso, K., Kinoshita, R. (1963). Absorption of sound wave by fabrics, Part 1: Absorption mechanism. Textile Machinery Society of Japan, 8(1), 32-39.

[21] Aso, K., Kinoshita, R. (1964). Absorption of sound wave by fabrics, Part 3: Flow resistance. Textile Machinery Society of Japan, 10(5), 236-241.

[22] Aso, K., Kinoshita, R. (1964). Sound absorption characteristicsof fiber assemblies. Textile Machinery Society of Japan, 10(5), 209-217.

[23] Na, Y. J., Lancaster, J., Casali, J., Cho, G. (2007). Sound absorption coefficients of micro-fiber fabrics by reverberation room method. Textile Research Journal, 77(5), 330-335.

[24] Lee, Y. E., Joo, C. W. (2003). Sound absorption properties of recycled polyester fibrous assembly absorbers. Autex Research Journal, 3(2), 77-84.
[25] Vassiliadis, S. (2012). Electronics and computing in textiles. (Bookboon).

[26] Yairi, M., Sakagami, K., Takebayashi, K., Morimoto, M. (2011). Excess sound absorption at normalincidence by two micro perforated panel absorbers with different impedance. Acoustical Science and Technology, 32(5), 194-200.

[27] Prasetiyo, I., Muqowi, E., Putra, A,, Novenbrianty, M., Desendra, G., Adhika, D. R., (2020). Modelling sound absorption of tunable double layer woven fabrics. Applied Acoustics, 157, 107008.

[28] Atalla, N., Sgard, F., Atalla, Y. (2005). On the modeling of perforated plates and screens. 12th ICSV, (Lisbon, July).

[29] Crocker, M. J., Arenas, J. P. (2007). Use of sound-absorbing materials. In: Crocker, M, J. (Ed.). Handbook of noise and vibration control. John Wiley \& Sons (New York).

[30] Greiner, W., Neise, L., Stocker, H. (1995). Thermodynamics and statistical mechanics. SpringerVerlag (New York). 